\title{
Teleosemantics Without Natural Selection
}

\author{
MARSHALL ABRAMS \\ Center for Philosophy of Biology, Box 90743, 201 West Duke Building, Duke University, \\ Durham, NC 27708, USA
}

Key words: belief, function, mental representation, naturalized intentionality, natural selection, teleology, wide content

\begin{abstract}
Ruth Millikan and others advocate theories which attempt to naturalize wide mental content (e.g. beliefs' truth conditions) in terms of function in the teleological sense, where a function is constituted in part by facts concerning past natural selection involving ancestors of a current entity. I argue that it is a mistake to base content on selection. Content should instead be based on functions which though historical, do not involve selection. I sketch an account of such functions.
\end{abstract}

\section{Introduction}

Like Ruth Millikan (1984, 1993b) I'm in favor of accounting for beliefs' contents, their truth conditions, in terms of natural teleology, and in particular in terms of functions which are constituted by past events. However, I'll argue that it's a mistake to account for content as Millikan does in terms of functions which are constituted by past natural selection. I'll go on to sketch a historical account of functions which does not depend on selection, arguing that this sort of function is a suitable basis for theory of content like Millikan's. Though the focus of my arguments will be on Millikan's theories of teleology and content, the points I make should apply to any theory of content according to which past natural selection plays some role in determining content.

Note that a theory of content such as Millikan's tries to give a naturalistic account of what it is for a belief to have a particular content. It does not try to explain the origin of beliefs or the mechanisms which produce them, except insofar as doing so helps to explain what content consists in. A theory of content provides a sort of reduction of beliefs' contents to naturalistically respectable features of the world (which, however, need not be viewed as being at a lower level than the semantic features to be reduced).

The primary purpose of this paper, then, is argue that natural selection is not part of what content $i s$. Where there is content, there was probably natural selection, but that fact is not part of what makes mental states contentful. 
Nevertheless, since I am sympathetic to teleological theories of content such as Millikan's, I want to explore the possibility of saving the general approach. My strategy is to try to define an alternative notion of function which does not depend on natural selection. Whether every challenge facing this alternative definition can be met remains to be seen, but it seems promising enough that it is worthy of development. While I will make clear that this alternative notion of function cannot fill common roles played by function concepts in biology, it may well be that it will have some utility outside of a theory of content.

In sections 1 and 2, I'll give a quick sketch of some of Millikan's teleological concepts and an even quicker sketch of parts of her theory of content. In sections 3 and 4 I'll argue that a theory of content should not be based on a historical theory of functions which involves selection. Section 5 will explain why I think that it nevertheless makes sense to base some common uses of "function" in biology on natural selection, and why theory of content is different from biology in this respect. Section 6 will sketch a "selectionless' definition of "ancestral history function" which I believe can provide a suitable basis for a theory of content.

\section{Teleology}

\subsection{Functions}

Millikan formulates her theory of content using a technical term which she defines, "proper function". I'll generally use "function" alone, context making clear when I intend Millikan's sense.

According to Millikan's account of functions, a function of my stomach, which contains the enzyme lactase, is to convert lactose (a component of milk) into other sugars. This is my stomach's function roughly because (1) some ancestors of mine were able to survive and reproduce partly as a result of converting lactose into other sugars, and (2) there were other people in some of my ancestors' populations who were less successful at having offspring because their stomachs did not contain lactase and couldn't digest milk very well.

Millikan's (1984, 1987, 1993a, 2002) basic definition of "proper function" can be viewed roughly as the claim that an object $o$ has causing events of type ${ }^{1}$ $F$ as its function if and only if three conditions are met.

First, in order for an item $o$ - say, my stomach - to have a function, it must have ancestors. I call this Millikan's "ancestor requirement". "Ancestor" is a technical term which Millikan defines with some care. For present purposes, the notion will become sufficiently clear if I illustrate it with some simple examples. To start with, "ancestor" applies to biological organisms in some- 
thing like the way that you'd expect: a parent is an ancestor of his or her children, children's children, and so on. ${ }^{2}$ Also, many parts of a parent, grandparent are ancestors of similar components of their children, grandchildren, etc. Thus my ancestors' stomachs are ancestors of my stomach. We can sidestep a few other technical terms by saying that something is always an ancestor of another thing with respect to some set of properties $p$, meaning that the two items share the properties $p$, where this sharing can be explained in certain ways. (This is a very loose gloss of what is covered fairly carefully in (Millikan 1984: Chapter 1).) Thus, those of my ancestors' stomachs which contained lactase and which are part of the reason that my stomach contains lactase are ancestors of my stomach with respect to containing lactase.

Second, in order for an item $o$ to have causing an event of type $F$ as its function, many of $o$ 's ancestors must have caused events of type $F$, and these $F$ events must have helped these ancestors of $o$ to reproduce - to help cause the creation of a similar item or items. Ultimately, perhaps after many generations, these other items must have produced $o$ itself. In other words, many of $o$ 's ancestors must have caused events of type $F$ which in turn helped to result in $o$ 's presence. Moreover, as a result of the ancestors causing these $F$ events, $o$ must be similar to these ancestors in having one or more of certain properties $p$ of the ancestors - namely a set of common properties which were often causally efficacious in causing events of type $F$. I call this second requirement for functionhood Millikan's "causal history requirement". For example, the causal history requirement will be satisfied for conversion of lactose to other sugars in my stomach, if lactase in many of my stomach's ancestors (my ancestors' stomachs) helped cause such a conversion, which in turn helped my ancestors and their stomachs to survive and reproduce because of the benefits of eating milk products in their environments.

Third, in order for $o$ to have a function, the items with the trait in question (the properties $p$ ) must have been selected for in contrast to other items lacking the trait, items which as a result of lacking the trait failed to cause $F$ events. More precisely, some ancestors of $o$ must have been members of a group of similar objects, some of which lacked the relevant properties $p$. These individuals which lacked $p$ must have in some sense been in competition for resources with those individuals which did have $p$. And the group members without $p$ must have had fewer descendants than did those with $p$ because, due to lacking $p$, these ' $p$-less' members failed to cause events of type $F$ as often as did those with $p$.

I'll summarize these three requirements:

Proper Function An item $o$ with one or more properties $p$ has causing events of type $F$ as its proper function if and only if:

$\mathbf{A R}$ (ancestor requirement) $o$ has ancestors with respect to $p$. 
And there were many past cases meeting the following requirements:

CHR (causal history requirement) An ancestor $a$ 's having properties $p$ led to the creation of later ancestors having $p$, and ultimately $o$, by causing events of type $F$.

SR (selection requirement) The ancestor $a$ must have had $p$-less competitors which failed to produce as many descendants on average as $a$, because, lacking $p$, the $p$-less competitors didn't cause events of the type $F$ as often as $a$.

\subsection{Normal conditions}

A normal condition (Millikan 1984, 1986, 1987, 1989) for performance of a function is a condition which is an essential part of explanations for the performance of the function, explanations that apply to most past cases of function performance by ancestors. Note that a normal condition may be relational. That is, a function may have a normal condition the specifics of which depend upon certain other conditions. Consider for example the rooting reflex exhibited by very young infants. If one of the infant's cheeks is touched, it 'roots', turning in the direction of that cheek while moving its mouth as during nursing. Presumably, the function of the rooting behavior is to help an infant find its mother's breast and thus to help cause nursing to occur. But it's crucial to explanations of most cases of this behavior leading to nursing that the mother's breast be in the direction of the touched cheek - whichever cheek it was. That this relation hold between brushed cheek and mother's breast is thus a normal condition for performance of the rooting reflex's function. We can call this a "relational normal condition", since the relevant condition is that a certain relation be satisfied.

Further, when a function has a relational normal condition, in any particular situation in which relata for the normal condition are tokened, I say that a function has an "instantiated relational normal condition" determined by the relational normal condition plus the relata. Thus if, say, an infant's right cheek is touched and the infant roots, an instantiated relational normal condition for the performance of the rooting behavior's function in that instance is that a mother's breast in fact be nearby to the infant's right.

\section{Mental content}

Consider a frog catching a small flying insect by suddenly extending its tongue to the insect's location and thus catching the insect. There is a mechanism in the frog which sometimes manages to track the location of a 
flying bug, and which thereby controls the extension of the tongue. (Exactly what I mean by "track" will be clarified below.) A signal of some sort is sent from the bug-tracking mechanism in the nervous system to motor-control mechanisms controlling the tongue, often causing the tongue to move to the location of a bug. It is presumably a function of the frog's tongue motorcontrol system to catch bugs, and this function has often been performed due to the action of the mechanism which tracks bug locations. ${ }^{3}$

Here we have an illustration of some of the basic ideas from Millikan's (1984, 1986, 1989, 1990a) theory of content. (Figure 1 may be useful as a visual summary of part of the following.) There is a 'producer' device, the bug-tracking mechanism ('bug-tracker') in the nervous system, which produces an 'indicative intentional icon', the signal sent to the motor-control mechanism. And there is a 'consumer' device, the tongue and its motorcontrol mechanism. (Call this the "tongue system".) Moreover, it is a function of the producer device, the bug-tracker, to help the consumer device, the tongue system, perform one of the latter's functions - in this case, catching a bug. This function of the producer is performed by means of the icon, the signal, being sent to the consumer. Usually, the way in which the icon helps the consumer device perform its function is by causing the consumer device to behave in a way that reflects certain facts about the world. The icon is able to do this because its form bears a certain relation ${ }^{4}$ to the state of the world - in particular, to the location of a bug. It is therefore a relational normal condition for the tongue system (consumer) performing its function that there be a bug in a location which is related in a certain way to the form of the signal. Thus when a particular signal is sent to the tongue system mechanism, it is an instantiated relational normal condition of the tongue system's function that there be a bug in a particular location. Finally, it is this instantiated relational normal condition which makes the content of this particular signal something like (the content of) "there's a bug in location $L$ now."

The preceding merely sketches certain aspects of the inner core of Millikan's theory of content; there is much that I must leave out here. Among other things, Millikan's full theory distinguishes the signals in the preceding example from beliefs, which satisfy additional requirements. Nevertheless, these signals have enough in common with beliefs according to the theory to illustrate the points I want to make. Without going into detail, let me add that in the case of a system with beliefs, the producer device will consist of those sensory and cognitive processes which give rise to and maintain beliefs, and the consumer device will consist of processes which lead to the fulfillment of desires. (Millikan offers a related account of the contents of desires.) 
102

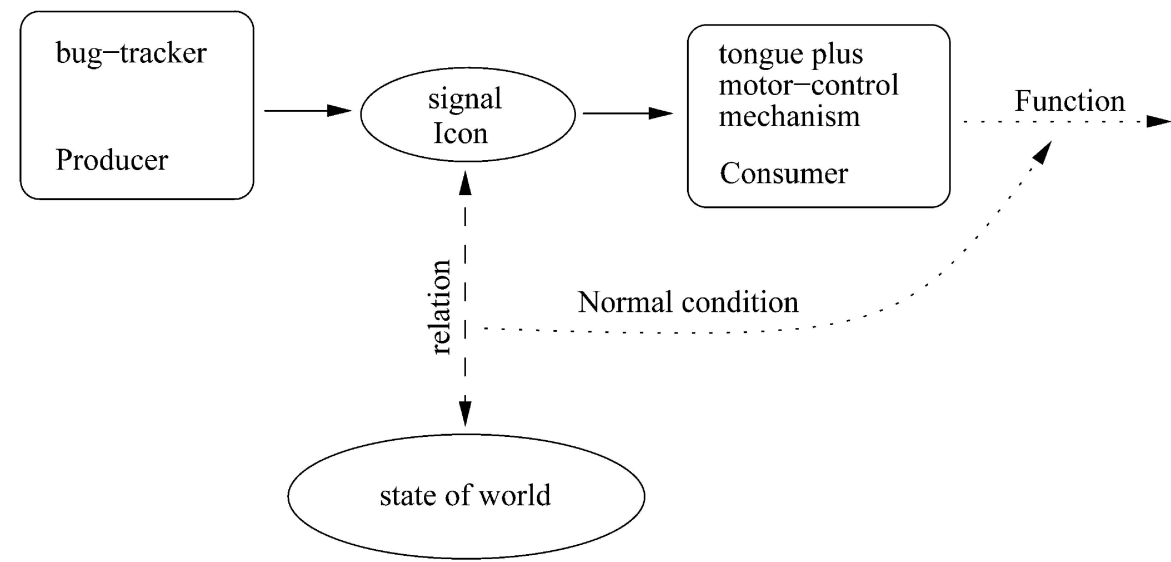

Figure 1. Some relationships in Millikan's theory of content.

\section{Selection as causally irrelevant}

Now I want to show that past selection need not have any causal consequences for an item which has a function. Thus, in requiring that parts of the cognitive system have certain functions, Millikan makes content depend on facts which may be causally irrelevant to the existence of the cognitive system. In section 4 I'll explain why I think this is a problem for Millikan's theory of content. In section 5 I'll make it clear why I do not think that this rules out the use of selection-based definitions of function in biology.

Here I'll describe two hypothetical scenarios: A situation in which selection occurs, and an alternative situation in which selection doesn't occur despite many causal facts remaining the same. In order to make my points as clear as possible, these scenarios are stripped down, simplified analogues of what may actually have occurred in nature. My goal here is to argue that a certain kind of occurrence (selection) is not part of what constitutes mental content. A simplistic scenario can help us to see just what conditions are essential to having content. Even if every cognitive system with contentful states is partly the result of natural selection, if it can be shown that there could have been content without selection, we will have learned something important about what content is. A more realistic example could have illustrated the same points, but the details of the example might obscure important aspects of the situation. Still, some readers may find it helpful if I indicate how the example I do give could be made more realistic, and I will do that at the end of this section.

The selection scenario. Suppose that in the past frogs survived by eating from a collection of nearby flying bugs, the frogs flicking their tongues into the 
air at random moments and thus sometimes catching food. Mutation then produced a frog that guided its tongue-flicking with the aid of a mechanism which was able to track the locations of bugs. Assume further that this new mutant frog was unable to flick its tongue as rapidly as earlier frogs. Let's make the example extremely simple: Assume that frogs reproduced asexually, and that soon after the new mutant frog appeared, the population contained only two frogs - Randy, the last of the old-fashioned random-flickers, and Guido, the new mutant guided-flicker.

Suppose that the air was thick enough with bugs close to Guido and Randy that random flicking was a workable strategy, but that nevertheless there were not so many bugs. Because Guido could catch bugs more readily, it repeatedly ate its fill before Randy could get enough to eat. Randy died without producing offspring, Guido had offspring, and the rest is (pre)history.

Assume that with its slower tongue, Guido would have failed to eat enough if it had only flicked randomly. Randy, with its stronger, more efficient tongue, had the capability to eat a sufficient number of bugs despite their relatively low density in the air, as long as something else - say, a frog with guided flicking - didn't eat too many of the bugs first. Note that Randy and Guido are in competition for a resource - bugs - and that Randy had fewer offspring than Guido because it lacked the guided-flicking trait. Thus selection against Randy's phenotype occurred.

Considering the catching of bugs by moving the tongue to a specific location as a possible function of Guido's motor-control mechanism, this is a case which satisfies both the causal history requirement, since catching bugs in this way helped cause reproduction, and the selection requirement, since Randy didn't have as many offspring. (In order to fully satisfy the requirements for the existence of a proper function, there would have to be a series of similar cases in subsequent or previous generations. For example, suppose that Guido's descendants repeatedly produced mutants without the guidedflicking trait, but that these frogs were selected against due to the presence of Guido's guided-flicking descendants.)

The no-selection scenario. Next the no-selection scenario: This case is similar to the selection scenario, but now Guido alone constitutes the entire population. Guido eats the same bugs as in the first case, has the same offspring, etc. But since there are no frogs which lack the guided-flicking trait, there can't be selection for the trait. Thus the selection requirement is not satisfied, and so this case cannot help make catching bugs by moving the tongue to a specific location into a proper function of Guido's descendant's tongues.

The important thing to notice is that the causal facts relevant to Guido's future and its descendants appear to be the same both in the selection and no- 
104

selection scenarios, but there can be content, according to Millikan, only in the selection scenario. According to Millikan's theory of functions, if Guido's descendants were never in situations like the selection scenario, a current frog's motor-control mechanism would not have catching bugs by moving the tongue to a specific location as a function. Recall that part of the reason that the signals sent to the motor-control mechanism had content according to Millikan's theory was that these signals were involved in a normal condition for the function of the mechanism. If the motor-control mechanism does not have catching bugs by moving the tongue to a location as its function, then there can be no normal conditions associated with the signals sent to the tongue mechanism, and thus the signals could not have content. But notice that the reason that Guido's bug-tracking mechanism does or doesn't have a function here is not anything that need affect the process leading from Guido up to the current item. Though Guido's presence affects Randy, Randy has no effect of any significance on Guido. (Randy's fate is merely a side-effect of Guido's presence.)

The Guido/Randy examples show that whether or not an item has a function at all may depend on the presence or absence of non-ancestors with no causal consequences for the item. But that also means that which functions an item has can depend on the same sort of peripheral facts. For example, suppose that the mechanism which allows Guido to guide its tongue toward bugs also sometimes guides the tongue so as to provide a healthful stretching of particularly tense mouth muscles. Suppose that Randy has a similar mechanism - that is, similar in its ability to usefully guide stretching, but dissimilar in its ability to help lead to eating. Then a Guido/Randy selection scenario could help constitute a bug-catching function but not a stretch-guidance function. Alternatively, suppose that Randy's mechanism is similar to Guido's in bug tracking but not in stretch guidance. Then a Guido/Randy selection scenario could only help to constitute a stretch-guidance function, not a bug-catching function. In the next section I'll explain why I think this is problematic given the role of functions in a teleological account of content; in section 5 I'll explain why I think it need not be problematic given the role of functions in biology.

As I mentioned above, the fact that the Guido/Randy example is unrealistic does not change its relevance to the goal of determining whether selection is part of what constitutes content. Nevertheless, some readers may find it helpful to consider ways in which the example might be made more realistic. Rather than two frogs, imagine a population in which the guided-flickers gradually proliferate. Rather than a single mutation, imagine a series of mutations, each leading to more precise but less efficient tongue placement. Since it's implausible that frogs would be limited to a small region in which bugs 
fly by, replace the population of frogs by a population of coral polyps in a narrow ocean trench; let some polyps acquire the ability to guide their tentacles toward prey.

\section{Content does not depend on selection}

We've seen that in order for the selection requirement to be satisfied with respect to a functional event type $F$, there must have been individuals - nonancestors, perhaps - in competition with ancestors, and these individuals had to have been worse at performing $F$ than ancestors. These individuals had to be worse at performing $F$ in the sense that because of their difference of character, they did not perform $F$ as often as the ancestors. But these nonancestor individuals did not have to help ancestors, hurt ancestors, affect ancestors' reproductive success, or affect ancestors or their descendants in any way. Nor did the non-ancestors have to have a tendency to affect any of these things in any way, be more likely to affect them, etc. The competitors could have simply been causally neutral with respect to the future of ancestors or their offspring (as Randy was in relation to Guido and its descendants). That is, these non-ancestors need not have had any causal relevance at all for the production of a current item whose possible function interests us. They can be causally inert in relation to the item. What happened to the competitors can be a mere side effect of the process leading up to the current item.

Yet the functions, normal conditions, and content of a thought can be determined by which of these non-ancestors happened to have existed. This, I feel, is a mistake. It seems a mistake to hold that the content of a thought, an aspect of an individual, is supposed to depend on processes and events which may have had no effect one way or another on the processes leading up to the individual. The contents of your thoughts could have differed, not because you or your ancestors had different characteristics, had a different degree of success or likelihood of success at reproducing, or lived in different environments. Your thoughts could have had different contents, or no contents at all, simply because of the presence or absence of individuals which were causally extraneous to you and the process that produced you. To avoid such consequences, a theory of content should not make content depend on whether there has been selection in the past nor on what kind of selection there has been in the past.

(There is a potential objection to the claim that selection can be causally irrelevant to the selected item, but it shouldn't bother us. Neander (1988, $1995 \mathrm{~b}, 1995 \mathrm{~d})$ and others have argued that selection sometimes explains the characteristics of individuals. I believe that Sober's (1995) and Walsh's (1998) arguments have shown that Neander's arguments do not apply to 
106

asexual species in which there is no recombination, but Neander might still be right about sexual species. ${ }^{5}$ Whether she is depends, I believe, on considerations which I won't elaborate here. But it seems to be an implication of Neander's arguments that in sexual species and a few others selection can sometimes have causal consequences for an item with a function. So if Neander's arguments succeed, would that show that content depends on selection after all? Certainly not. At best Neander's arguments would show that selection could be relevant to content some of the time in sexual species and some other species. But surely mental content should not depend on whether members of a species engage in sexual reproduction. That selection might be causally relevant to contentful cognitive systems in some cases is not enough to make it part of what constitutes content.)

\section{Biological functions can depend on selection}

Although I do not think that content is constituted by selection-based functions, content seems relatively special in needing some other sort of functions as its basis. Here very briefly is why I think it's likely that selection-based functions do have a role in biology.

Much of biology is explicitly concerned with populations and with changes in frequencies of types in populations. Selection is one kind of fact which explains changes of frequencies of types in populations. As I explain below in section 6.1, a common view is that selection occurs only when one genotype or phenotype is fitter than another. Relative fitness is often considered a relationship between two alternative types and some characterization of probable success, for example expected number of offspring. But talk of relative fitness does not by itself tell us much about the causal processes that were responsible for the difference in the alternative traits' probable reproductive success. Talk of functions can be a way of filling in some of the missing detail by specifying what sort of effect of a fitter trait it is that's responsible for its greater fitness. The function of a trait in this sense would be that kind of effect which the trait caused more often, more effectively, etc. than alternative traits, where performance of the function (more often, effectively, etc.) is what is responsible for the difference in probable reproductive success. (A particular version of this picture is spelled out in section 6.1.) In such a context it's beside the point to complain that nonancestors who were selected against have no causal consequences for a single current item. What's at issue is the composition of the population and why it changed, and selection plausibly is causally relevant to this. In this context a selection-based theory of functions might be just what's called for. Note 
that function talk may also be used in other ways that do not have to do with history. ${ }^{6}$

\section{Historical functions without selection}

One virtue of a theory of functions like Millikan's is that since it is only past events which constitute proper functions, a current item need not perform these functions. Similarly, in part because normal conditions are constituted by past events, a function may be performed without its normal conditions obtaining. Thus since the truth-conditions of a belief are constituted in part by a relational normal condition for a function, the fact that an aspect of a cognitive system has that function with that normal condition does not imply that these truth-conditions are met. More specifically, in a particular situation the instantiated relational normal condition for performance of a belief's consumer's function is a condition which need not obtain. We can summarize the point this way: Content is defined in terms of relationships which held in the past. A belief is true if and only if these relationships hold now. But they need not hold. This allows for the possibility that beliefs can be either true or false, which is an essential desideratum for a theory of content. Thus it's plausible that the fact that functions and normal conditions are defined in terms of history will allow a theory of content based on them to solve what Fodor $(1990,1984)$ labeled the "disjunction problem".

So though it's problematic to base content on past natural selection, it's reasonable to base content on past events of some sort. We can retain the virtues of Millikan's theory of content by defining a notion of function in terms of past events which involve ancestors but no non-ancestors, using this special kind of a function as the basis of a theory content. In what follows I will sketch such a notion of function, which I call "ancestral history function" (§6.2). My aim here will be only to point in the direction of a full-fledged account of ancestral history functions. A more detailed discussion of this kind of function can be found in (Abrams 2002: Chapter 9).

To see what's behind the idea of an ancestral history function, note that there's a natural intuition that a trait with a function is one which in some sense has improved the lot of organisms with that trait - one that has in some sense made organisms better than they would have been. Here "better" can mean "better at reproduction." But better than what? Selection requirements are based on the idea that a functional trait makes an organism better at reproduction than an alternative trait present in an ancestral population. Note, though, that an organism with a function-supporting trait is typically also better at reproduction than some of its own ancestors, or at least better than they would have been in its current environment. ${ }^{7}$ It is this idea 
which is behind the notion of an ancestral history function. The rationale behind the definition of "ancestral history function" will be clearer, however, if we first look at a refinement of Millikan's selection requirement, one which includes elements which will reappear in the ancestral history function definition.

\subsection{A refined selection requirement}

First note that if we say that there is selection for types which are "better at reproduction," this cannot mean simply "has more offspring," or we will not be able to understand the distinction which biologists make between natural selection and drift. Drift is change in the composition of a population purely due to chance, while selection is change in the composition of a population which is at least partly due to some types of organisms in the population being fitter than others. But note that if "fitter" meant "has more offspring," drift could not be distinguished from selection. The popular though not uncontroversial 'propensity interpretation of fitness' resolves this problem by defining a type's (genotype's or phenotype's) fitness as its expected number of offspring. That is, fitness is the weighted average of all possible numbers of offspring, where the weights are objective probabilities of that type having a certain number of offspring in a given environment. ${ }^{8}$ In what follows I will take for granted that some conception of fitness like this is correct.

A selection requirement will best accord with this picture if the fact that $F$ is a trait's function implies that there are objective probabilities (probabilistic laws) which explain why the trait caused $\mathrm{F}$ and why performing $F$ improved fitness and thus led to the trait being selected over others. Here is such a selection requirement:

$\mathbf{S R}^{\prime}$ (selection requirement with probabilistic refinements) In addition to the ancestor $a$, with properties $p$, there must have been some similar competitors with an alternative set of properties $p^{\prime}$ such that

1. (a) the competitors with $p^{\prime}$ failed to produce as many descendants as $a$ on average, because

(b) $p$ is fitter than $p^{\prime}$,

and this is so because

2. (a) $a$ caused $F$ more often (or in more appropriate circumstances, etc.) on average than did the competitors with $p^{\prime}$, because

(b) the probability of $F$ being caused (in appropriate circumstances, etc.) by an item with $p$ is higher than that for an item with $p^{\prime}$, 
3. (a) $a$ had more offspring on average than other similar organisms which didn't cause $F$ as often (etc.) because

(b) it is fitter to perform $F$ more often (or in more appropriate circumstances, etc.) than to perform it less often (etc.). ${ }^{9}$

Each of the three numbered parts of $\mathrm{SR}^{\prime}$ consists of a clause (a) which says what caused what, and a clause (b) which gives a probabilistic generalization which, roughly speaking, governs the causal interactions in question. (Remember that I am taking fitness to be reducible to certain probabilistic facts.) Part 1 merely says that selection took place, expressing the claim in terms of relationships between the traits $p$ and $p^{\prime}$ and reproduction. Parts 2 and 3 explain the latter relationships in terms of relationships between the traits and function performance (2), and relationships between function performance and reproduction (3). ${ }^{10}$

$\mathrm{SR}^{\prime}$ makes it clear that being better at reproduction is not simply accidentally having more offspring. The (b) clauses make it clear that neither $F$ being performed more often nor $F$ causing more offspring to be produced is accidental. These effects occur more often because objective probabilities are such as to make them more likely to occur more often. It is the (b) clauses which will be at the core of the notion of an ancestral history function, allowing a distinction between real ancestral history functions and merely accidental effects.

\subsection{Ancestral history functions}

If an organism $a$ has a novel trait, then rather than comparing $a$ 's fitness and probability of function performance, etc. with those of competitors, we can make essentially the same comparison with $a$ 's parent. We can incorporate these comparisons into a requirement something like $\mathrm{SR}^{\prime}$, and use that as the basis of the definition of function ("ancestral history function") which will make reference only to ancestors of a current item. A teleological theory of content based on this kind of function will then plausibly avoid the problem presented above in section 4.

The analogue of SR' in definition of "ancestral history function" is the 'near ancestor nomic comparison requirement' (NAR), which is built around the probability and fitness clauses $1 b, 2 b$, and $3 b$ from $S^{\prime}$; we can use the same wording to compare with respect to an earlier ancestor rather than competitors. An ancestor requirement AR and causal history requirement CHR are copied directly from the definition of "proper function" in Section 1.1. Letting "near ancestor" mean an ancestor which is no more than a few generations back from an item, here is a definition of "ancestral history function" (a simple version, appropriate for simple cases): ${ }^{11}$ 
Ancestral History Function An item $o$ with one or more properties $p$ has causing events of type $F$ as its ancestral history function if and only if:

$\mathbf{A R}$ (ancestor requirement) $o$ has ancestors with respect to $p .^{12}$

And there were many past cases meeting the following requirements:

CHR (causal history requirement) An ancestor $a$ 's having properties $p$ led to the creation of later ancestors having $p$, and ultimately $o$, by causing events of type $F$.

NAR (near ancestor nomic comparison requirement)

The ancestor $a$ (with properties $p$ ) had a near ancestor with an alternative set of properties $p^{\prime}$ such that

1. $p$ is fitter than $p^{\prime}$, and this is so because:

2. the probability of $F$ being caused (in appropriate circumstances, etc.) by an item with $p$ is higher than that for an item with $p^{\prime}$,

and

3. it is fitter to perform $F$ more often (or in more appropriate circumstances, etc.) than to perform it less often (etc.). ${ }^{13}$

Clause 1 of NAR requires that the functional trait be fitter than its near ancestor, and clauses 2 and 3 require that this increase in fitness be due to an increase in the probability of causing an effect which increases fitness. It is too much to ask, and it is unnecessary, to include in NAR requirements parallel to SR's 1a, 2a, and 3a. Note that without NAR we would be left with a notion of function based only on the ancestor requirement $\mathrm{AR}$ and the causal history requirement CHR. A function defined in this way might be constituted by a series of unlikely occurrences which would seem to have nothing to do with anything like functions. ${ }^{14}$

Functional traits do not, in general, spring into existence suddenly through a mass of coordinated mutations. They arise gradually through a long series of changes in the genetic code of a lineage. A complete definition of "ancestral history function" must be able to account for the ancestral history function of a current item in terms of a series of comparisons between traits and near ancestors, each representing an incremental change toward the current trait with its current function. (Abrams 2002: §§9.2.1, 9.3.5) offers a way of handling this issue. (Selection-based accounts of function face this problem as well, since competition in a population is usually between merely incrementally different traits.)

Note that though earlier traits are screened off from later traits by intermediate traits, this relationship is typical of causal chains. The problem with a 
selection-based theory of content is that it allows content to depend on events which may not be part of any causal chain of events leading to the system with contentful states; an ancestral history based theory of content does not have this problem.

\subsection{Ancestral history function as a basis of content}

My objection in section 4 to making a theory of content depend on selection was based on the intuition that content should not be changed by past items with no causal consequences for the present item with beliefs. But ancestral history functions are defined solely in terms of ancestors, that is, in terms of past entities which do have causal consequences for the present item. Thus ancestral history functions, unlike proper functions, are a suitable basis for a theory of content like Millikan's.

Consider the no-selection scenario in which Guido alone existed. Since Guido did not compete with any frogs which lacked the guided-flicking trait, that trait's effects did not satisfy Millikan's selection requirement, and Guido's successful reproduction did not help to constitute flick-guiding signals' content in Guido's descendants. But recall that Guido's guidedflicking trait was the result of a new mutation; Guido's parent lacked this trait. Let us assume that Guido's parent was just like the frog Randy in the selection scenario. The no-selection scenario then satisfies NAR, the nearancestor nomic comparison requirement: Guido, with properties $p$ - the guided-flicking trait - had a near ancestor, the parent, with an alternative set of properties $p^{\prime}$ - the random-flicking trait - where the following holds: (1) The guided-flicking trait is fitter than the random-flicking trait because, for example, the probability of surviving is greater for an individual with guidedflicking trait than for one with the random-flicking trait. This is so because of the following: (2) Having the guided-flicking trait raises the probability of catching bugs by moving the tongue to a specific location; in fact, in this example, the probability of this occurring given the alternative, randomflicking trait is zero. (3) In Guido's environment, it is fitter to catch bugs by moving the tongue to a specific location than by flicking randomly. Thus NAR is satisfied. CHR, the causal history requirement is satisfied as well, as long as eating bugs by moving the tongue to specific locations helps Guido to survive and reproduce. Thus the no-selection scenario could count as one of the "many past cases" which help to constitute an ancestral history function for some perhaps distant descendant of Guido's. Note that the selection scenario could also satisfy CHR and NAR, still helping to constitute content for Guido's descendants. If we base a Millikan-style theory of content on ancestral history functions, content will not be affected by whether ancestors of the contentful system have non-ancestral competitors. 
Moreover, as with selection-based functions, the performances which constitute an ancestral history function are in the past. Ancestral history functions need not be performed in the present, and if they are performed, it need not be due to normal conditions obtaining. An intentional icon such as a tongue-control signal can be tokened without normal conditions for the consumer's ancestral history function obtaining. Thus ancestral history functions allow misrepresentation.

\section{Conclusion}

Of two alternative types, one is reproduced and the other is not. That is the relation at the heart of natural selection. But this relation between types does not require that the type selected against interact with the type selected, nor with the latter's descendants. This is why it is that if content is based on teleology, and teleology on selection, then part of what determines content needn't have a causal connection to the contentful states.

If this is a problem for Millikan's theory of content, it would seem to be a problem for any selection-based theory of content. The point applies to theories of content like Neander's (1995c) and Sterelny's (1990) which give selection a relatively limited role in determining content, as well as to theories like Millikan's and Papineau's $(1984,1987)$ which give selection a larger role in determining content. The point applies to Dretske's (1988) account of content in terms of learning treated as a kind of natural selection. (Millikan and Papineau allow learning to provide a basis for functions and content, too.)

In general, it's plausible that where there are selection-based functions, there are usually corresponding ancestral history functions, and vice versa. An improvement in a cognitive system tracking the world often arises as modification of an earlier trait which does not allow tracking the world so well. Such incidents can help to constitute ancestral history functions, but such incidents will usually be ones in which there are other instances of the earlier trait in the population, so there will be selection against the earlier trait, too. Thus the trait will have a corresponding selection-based function as well. A similar argument can be given that where there is selection, NAR is usually satisfied. Thus, apart from a few unusual cases involving small populations or selection for lower fitness (see note 7), a theory of content based on organismic selection will do the same work when revised to use ancestral history functions, albeit without taking content to depend on selection.

That seems reasonable for selection on organisms, though it's not clear whether ancestral history functions can replace selection-based functions in theories of content in terms of learning. If it can be argued that in learning 
there are analogues of probabilities of function performance and of differences in fitness due to differences in function performance, then perhaps a variation on ancestral history function would allow a reasonable revision of such a theory of content. ${ }^{15}$

In this paper I've raised what appears to be a problem for any selectionbased theory of content. I've offered a solution for theories of content based on organismic selection. Whether the same solution might resolve the problem for theories of content based on other kinds of selection remains to be seen.

\section{Acknowledgements}

I'm very grateful for comments from Kim Sterelny and two anonymous reviewers, and for comments on this paper's ancestors by Ruth Millikan, Bill Wimsatt, and Murat Aydede.

\footnotetext{
Notes

1 In this paper I use "event", "condition", "fact", and "situation" unsystematically to refer to things which can enter into causal interactions. Ultimately I think such language needs to be regimented a bit, but I do not do so here. In any event, whatever ontology of causal relata we end up with, I think it likely that when a causal interaction takes place, it does so in virtue of properties of something - events, or objects, perhaps. When I talk of the type of an event, I mean to refer to those properties relevant to whether certain causal interactions take place or would take place.

2 It appears that older siblings, aunts and uncles, great-aunts, etc., also count as ancestors in Millikan's sense, but this fact will not much matter here. See (Millikan 1984: Chapter 1), especially clause (2) of the definition of "ancestor" on p. 27.

3 For details of how some of this works in actual frogs and toads, see for example (Ewert 1974) and (Lettvin et al. 1959).

4 For details concerning this relation see especially (Millikan 1984: Chapter 6).

5 (McClamrock 1993), (Millikan 1990b), and (Matthen 1999) also argue that selection can explain characteristics of individuals; (Cummins 1975), (Dretske 1988), (Dretske 1990), and (Pust 2001) argue that it does not.

6 See for example (Cummins 1975), (Bigelow and Pargetter 1987), (Millikan 2002).

7 What if significant aspects of our cognitive system arose as mutations from traits with higher fitness, and these aspects of the cognitive system were nevertheless selected for because their presence lowered the fitness of competitors? It could turn out then that these aspects of the cognitive system have biological functions but not ancestral history functions. Then my view could imply that none of our mental states have content, which is to say that what I am advocating is not, it seems, a theory of content. However, it's implausible that changes in cognitive systems which gave rise to content according to Millikan's theory lowered fitness relative to earlier traits. Millikan's account of content depends on states in the cognitive system sometimes paralleling aspects of the state of the world and thereby helping an organism to
} 
coordinate its behavior with the state of the world. Making behavior sensitive to the state of the world is surely generally useful, and mutations which make behavior more sensitive to states of the world surely have often increased fitness.

8 See for example (Brandon 1978) or (Mills and Beatty 1979) for the simple propensity interpretation of fitness described here. (Beatty and Finsen 1989) and (Brandon 1990) modify the propensity interpretation of fitness, making fitness a more complex function of probabilities. Although I do not believe that fitness can be defined in terms of propensity, as the propensity interpretation of fitness claims, I do think that fitness should be defined in terms of some kind of objective probability (Abrams 2003). Ideas which might form the basis of a replacement for propensity in an account of fitness can be found in (Abrams 2000), (Abrams 2002), (Strevens 2003), (Miller 1995: 141f), (Millikan 2001), and (Weber 2001).

9 If we replace Millikan's selection requirement SR with $\mathrm{SR}^{\prime}$, we get an account of functions similar to Neander's (1991), which reads:

It is the/a proper function of an item $(X)$ of an organism $(O)$ to do that which items of $X$ 's type did to contribute to the inclusive fitness of $O$ 's ancestors, and which caused the genotype, of which $X$ is the phenotypic expression, to be selected by natural selection. (Neander 1991: 174)

The SR' modification of Millikan's definition differs from Neander's most significantly in that it (a) spells out a sense of "contribute", (b) does not make use of the genotype/phenotype distinction and the concept of inclusive fitness, and (c) makes it explicit that it is differences in fitness and in "contributing" that resulted in selection. The patterns which Neander captures with (b) can be handled by the SR'-based definition using details of Millikan's concept of "ancestor" which are not presented in this paper.

$10 \mathrm{SR}^{\prime}$ is a very simple requirement which ought to be generalized in certain ways; it ignores many issues. It only fits with the simple version of the propensity interpretation of fitness according to which a type's fitness is its expected number of offspring. It is vague about whether it is useful to perform a function more times rather than fewer times, whether there are diminishing returns to performing it too often, what other factors affect the probabilities of reproduction given function performance, etc. A more careful discussion of some of these issues can be found in (Abrams 2002: Chapter 9).

11 Some aspects of this definition are based on insights gleaned from Wimsatt's discussion of function in (Wimsatt 1972). The initial intuition motivating development of this definition probably derives from ideas in (McClamrock 1993).

12 Technically, Millikan's concept of an ancestor may not be what we want here. As I mentioned in note 2, an ancestor in Millikan's sense could actually be an older sibling, for example. A more restricted sense of "ancestor" might be more appropriate for the definition of "ancestral history function".

13 It's arguable that ancestral history functions are instances of the general etiological sense of function which Larry Wright $(1973,1976)$ characterized. Roughly, to say that a type $X$ has $Z$ as its ancestral history function is to say that in the past $X$ doing $Z$ raised the probability of a subsequent $X$ existing, compared to the probability relative to $X$ 's near ancestor which did not do $Z$. That is close to saying that $X$ is here because it did $Z$. It is to say that $X \operatorname{doing} Z$ helped later instances of $X$ to be here, i.e. that $Z$ increased the probability that an $X$ would be here.

14 For example, suppose that a crawling insect had an unpredictable internal state $L$, which when tokened caused the insect to turn left. Suppose that, just by chance, the insect thereby repeatedly avoided predators who happened to be on the right when $L$ was tokened. Repeated cases like this could lead to $L$ having the function of causing left turns, and even having as its 
content that a predator is to the right. This would be so even though there is nothing systematic about the environment which makes turning left adaptive.

15 There need not be ancestral history functions generated directly by the learning process in order to attribute a derivative kind of ancestral history function to the outcomes of that process. Millikan (1984: Chapter 2) defines senses of "function" - adapted proper function and derived proper function - whose performance need not have been part of the history leading up to to the item with the function. If a particular learning process is the product of a mechanism which has as its function producing such processes, that process may have adapted or derived functions because of selection for that mechanism, and likewise for the product of the learning process. But adapted or derived functions can just as well be based on ancestral history functions, and these derivative senses of function may apply to learning processes even if the processes are not by themselves able to constitute ancestral history functions.

\section{References}

Abrams, M.: 2000, 'Short-Run Mechanistic Probability'. Presented at the Philosophy of Science Association, Seventeenth Biennial Meeting, Vancouver B.C., Canada, November $2-5,2000$.

Abrams, M.: 2002, 'Probabilistic Foundations of Teleology and Content'. Ph.D. thesis, University of Chicago.

Abrams, M.: 2003, 'Is Fitness a Function of Propensity?', Unpublished MS.

Beatty, J. and Finsen, S.: 1989, 'Rethinking the Propensity Interpretation: A Peek Inside Pandora's Box', in M. Ruse (ed.), What the Philosophy of Biology is, Kluwer Academic Publishers, pp. 17-30.

Bigelow, J. and Pargetter, R.: 1987, 'Functions', Journal of Philosophy 84(4), 181-196.

Brandon, R.: 1978, 'Adaptation and Evolutionary Theory', Studies in the History and Philosophy of Science 9(3), 181-206. Reprinted in (Sober 1984).

Brandon, R.: 1990, Adaptation and Environment, Princeton University.

Cummins, R.: 1975, 'Functional Analysis', Journal of Philosophy 72(20), 741-764. Reprinted in (Sober 1984) and (Sober 1994).

Dretske, F.: 1988, Explaining Behavior, MIT Press.

Dretske, F.: 1990, 'Reply to Reviewers', Philosophy and Phenomenological Research L(4), 819-839.

Ewert, J.-P.: 1974, 'The Neural Basis of Visually Guided Behavior', Scientific American 230(3), 34-42.

Fodor, J.: 1984, 'Semantics, Wisconsin Style', Synthese 59, 231-250.

Fodor, J.: 1990, 'Psychosemantics, or: Where Do Truth Conditions Come From?', in W.G. Lycan (ed.), Mind and Cognition, Basil Blackwell, pp. 312-337. This paper was widely circulated in manuscript form during the 1980s.

Lettvin, J., Maturana, U., McCulloch, W. and Pitts, W.: 1959, 'What the Frog's Eye Tells the Frog's Brain', Proceedings of the IRE, pp. 1940-1951.

Matthen, M.: 1999, 'Evolution, Wisconsin Style: Selection and Explanation of Individual Traits', British Journal for the Philosophy of Science 50, 143-150.

McClamrock, R.: 1993, 'Functional Analysis and Etiology', Erkenntnis 38(2), 249-260.

Miller, D.W.: 1995, 'Propensities and Indeterminism', in A. O'Hear (ed.), Karl Popper: Philosophy and Problems, Cambridge University Press.

Millikan, R.G.: 1984, Language, Thought, and Other Biological Categories, MIT Press. 
Millikan, R.G.: 1986, 'Thoughts without Laws; Cognitive Science With Content', Philosophical Review 95(1), 47-50. Reprinted in (Millikan 1993b).

Millikan, R.G.: 1987, 'In Defense of Proper Functions', Philosophy of Science 56(2), 288302. Reprinted in (Millikan 1993b).

Millikan, R.G.: 1989, 'Biosemantics', Journal of Philosophy 86(6), 281-297. Reprinted in (Millikan 1993b).

Millikan, R.G.: 1990a, 'Compare and Contrast Dretske, Fodor, and Millikan on Teleosemantics', Philosophical Topics 18(2), 151-161. Reprinted in (Millikan 1993b).

Millikan, R.G.: 1990b, 'Seismograph Readings for "Explaining Behavior"', Philosophical and Phenomenological Research L(4), 807-812.

Millikan, R.G.: 1993a, 'Propensities, Exaptations, and the Brain', in (Millikan 1993b), Chapter 2, pp. 31-50.

Millikan, R.G.: 1993b, White Queen Psychology and Other Essays for Alice, MIT Press.

Millikan, R.G.: 2001, 'What has Natural Information to Do with Intentional Representation?', in D. Walsh (ed.), Naturalism, Evolution and Mind, Cambridge University Press.

Millikan, R.G.: 2002, 'Biofunctions: Two Paradigms', in A. Ariew, R. Cummins and M. Perlman (eds.), Functions: New Essays in the Philosophy of Psychology and Biology, Oxford University Press.

Mills, S. and Beatty, J.: 1979, 'The Propensity Interpretation of Fitness', Philosophy of Science 46(2), 263-286. Reprinted in (Sober 1984) and (Sober 1994).

Neander, K.: 1988, 'Discussion: What Does Natural Selection Explain? Correction to Sober', Philosophy of Science 55, 442-426.

Neander, K.: 1991, 'Functions as Selected Effects: The Conceptual Analyst's Defense', Philosophy of Science 58, 168-184.

Neander, K.: 1995b, 'Explaining Complex Adaptations: A Reply to Sober's "Reply to Neander" ', British Journal for the Philosophy of Science 56, 583-587.

Neander, K.: 1995c, 'Misrepresenting \& Malfunctioning', Philosophical Studies 79, 109-141.

Neander, K.: 1995d, 'Pruning of Tree of Life', British Journal for the Philosophy of Science 46, 59-80.

Papineau, D.: 1984, 'Representation and Explanation', Philosophy of Science 51, 550-572.

Papineau, D.: 1987, Reality and Representation, Basil Blackwell.

Pust, J.: 2001, 'Natural Selection Explanation and Origin Essentialism', Canadian Journal of Philosophy 31(2), 201-220.

Sober, E. (ed.): 1984, Conceptual Issues in Evolutionary Biology, MIT Press.

Sober, E. (ed.): 1994, Conceptual Issues in Evolutionary Biology, 2nd ed. MIT Press.

Sober, E.: 1995, 'Natural Selection and Distributive Explanation: A Reply to Neander', British Journal for the Philosophy of Science 46, 384-397.

Sterelny, K.: 1990, The Representational Theory of Mind: An Introduction, Basil Blackwell.

Strevens, M.: 2003, Bigger Than Chaos: Understanding Complexity through Probability, Harvard University Press.

Walsh, D.M.: 1998, 'The Scope of Selection: Sober and Neander on What Natural Selection Explains', Australasian Journal of Philosophy 76(2), 250-264.

Walsh, D.M.: 2001, 'Determinism, Realism, and Probability in Evolutionary Theory: The Pitfalls, and How to Avoid Them', Philosophy of Science 68(3), S213-S224.

Wimsatt, W.C.: 1972, 'Teleology and the Logical Structure of Function Statements', Studies in History and Philosophy of Science 3(1), 1-80.

Wright, L.: 1973, 'Functions', Philosophical Review 82, 139-168. Reprinted in (Sober 1984) and (Sober 1994).

Wright, L.: 1976, Teleological Explanations, University of California Press. 